\title{
Geodesic Lightlike Submanifolds of Indefinite Sasakian Manifolds*
}

\author{
Junhong Dong ${ }^{1}$, Ximin Liu ${ }^{2}$ \\ ${ }^{1}$ Department of Mathematics, South China University of Technology, Guangzhou, China \\ ${ }^{2}$ School of Mathematical Sciences, Dalian University of Technology, Dalian, China \\ E-mail: dongjunhong-run@163.com,ximinliu@dlut.edu.cn \\ Received July 23, 2011; revised August 15, 2011; accepted August 25, 2011
}

\begin{abstract}
In this paper, we study geodesic contact CR-lightlike submanifolds and geodesic screen CR-lightlike (SCR) submanifolds of indefinite Sasakian manifolds. Some necessary and sufficient conditions for totally geodesic, mixed geodesic, $\bar{D}$-geodesic and $D^{\prime}$-geodesic contact CR-lightlike submanifolds and SCR submanifolds are obtained.
\end{abstract}

Keywords: CR-Lightlike Submanifolds, Sasakian Manifolds, Totally Geodesic Submanifolds

\section{Introduction}

A submanifold $M$ of a semi-Riemannian manifold $\bar{M}$ is called lightlike submanifold if the induced metric on $M$ is degenerate. The general theory of a lightlike submanifold has been developed by Kupeli [1] and BejancuDuggal [2].

The geometry of CR-lightlike submanifolds of indefinite Kaehler manifolds was studied by Guggal and Bejancu [2]. The geodesic CR-lightlike submanifolds in indefinite Kaehler manifolds were studied by Sahin and Günes [3,4].

Lightlike submanifold of indefinite Sasakian manifolds can be defined according to the behavior of the almost contact structure, and contact CR-lightlike submanifolds and screen CR-lightlike (SCR) submanifolds of indefinite Sasakian manifolds were studied by Duggal and Sahin in [5]. The study of the geometry of submanifolds of indefinite Sasakian manifolds has been developed by [6] and others.

In this paper, geodesic contact CR-lightlike submanifolds and geodesic screen CR-lightlike (SCR) submanifolds of indefinite Sasakian manifolds are considered. Some necessary and sufficient conditions for totally geodesic, mixed geodesic, $\bar{D}$-geodesic and $D^{\prime}$-geodesic contact CR-lightlike submanifolds and SCR submanifolds are obtained.

"This work is supported by NSFC (10931005).

\section{Preliminaries}

A submanifold $M^{m}$ immersed in a semi-Riemannian manifold $\left(\bar{M}^{m+n}, \bar{g}\right)$ is called a lightlike submanifold if it admits a degenerate metric $g$ induced from $\bar{g}$ whose radical distribution RadTM is of rank $r$ where $1 \leq r \leq m, \operatorname{Rad} T M=T M \cap T M^{\perp}$, where

$$
T M^{\perp}=\bigcup_{x \in M}\left\{u \in T_{x} \bar{M} \mid \bar{g}(u, v)=0, \forall v \in T_{x} \bar{M}\right\} .
$$

Let $S(T M)$ be a screen distribution which is semiRiemannian complementary distribution of RadTM in $T M$, i.e. $T M=\operatorname{RadTM} \perp S(T M$. As $S(T M)$ is a nondegenerate vector subbundle of $\left.T \bar{M}\right|_{M}$, we put $\left.T \bar{M}\right|_{M}=S(T M) \perp S(T M)^{\perp}$.

We consider a nondegenerate vector subbundle of $S(T M)$, which is a complementary vector bundle of RadTM in $T M^{\perp}$. Since, for any local basis $\left\{\xi_{i}\right\}$ of RadTM , there exists a local frame $\left\{N_{i}\right\}$ of sections with value in the orthogonal complement of $S\left(T M^{\perp}\right)$ such that $\bar{g}\left(\xi_{i}, N_{i}\right)=\delta_{i j}$ and $\bar{g}\left(N_{i}, N_{j}\right)=0$, there exists a lightlike, transversal vector bundle $\operatorname{ltr}(T M)$ locally spanned by $\left\{N_{i}\right\}$. Let $\operatorname{tr}(T M)$ be the complementary (but not orthogonal) vector bundle to $T M$ in $\left.T \bar{M}\right|_{M}$.

Then

$$
\begin{gathered}
\operatorname{tr}(T M)=\operatorname{ltr}(T M) \perp S\left(T M^{\perp}\right), \\
T \bar{M}=S(T M) \perp[\operatorname{Rad} T M \oplus \operatorname{ltr}(T M)] \perp S\left(T M^{\perp}\right) .
\end{gathered}
$$


Now, let $\bar{\nabla}$ be the levi-Civita connection on $\bar{M}$, we have

$$
\begin{aligned}
& X(\bar{g}(Y, Z))=\bar{g}\left(\bar{\nabla}_{X} Y, Z\right)+\bar{g}\left(Y, \bar{\nabla}_{X} Z\right), \\
& \forall X, Y, Z \in \Gamma(T M), \\
& \bar{\nabla}_{X} Y=\nabla_{X} Y+h(X, Y), \forall X, Y \in \Gamma(T M), \\
& \bar{\nabla}_{X} V=-A_{V} X+\nabla_{X}^{t} V, \forall X \in \Gamma(T M), \\
& V \in \Gamma(\operatorname{tr}(T M)),
\end{aligned}
$$

where $\left\{\nabla_{X} Y, A_{V} X\right\}$ and $\left\{h(X, Y), \nabla_{X}^{t} V\right\}$ belong to $\Gamma(T M)$ and $\Gamma(\operatorname{tr}(T M))$,

respectively. Using the projectors

$l: \operatorname{tr}(T M) \rightarrow S(T M)$ and $s: \operatorname{tr}(T M) \rightarrow \operatorname{ltr}\left(T M^{\perp}\right)$, from [1], we have

$$
\begin{array}{r}
\bar{\nabla}_{X} Y=\nabla_{X} Y+h^{l}(X, Y)+h^{s}(X, Y), \forall X, Y \in \Gamma(T M), \\
\bar{\nabla}_{X} N=-A_{N} X+\nabla_{X}^{l} N+D^{s}(X, N), \forall N \in \Gamma(\operatorname{ltr}(T M)), \\
\bar{\nabla}_{X} W=-A_{W} X+\nabla_{X}^{s} W+D^{l}(X, W), \forall W \in \Gamma\left(S\left(T M^{\perp}\right)\right) .
\end{array}
$$

Denote the projection of $T M$ to $S(T M)$ by $P$, we have the decomposition

$$
\begin{gathered}
\nabla_{X} P Y=\nabla_{X}^{*} P Y+h^{*}(X, P Y), \\
\nabla_{X} \xi=-A_{\xi}^{*} X+\nabla_{X}^{* t} \xi,
\end{gathered}
$$

for any $X, Y \in \Gamma(T M), \xi \in \Gamma(\operatorname{RadTM}), N \in \Gamma(\operatorname{ltr}(T M))$. From the above equations we have

$$
\begin{gathered}
\bar{g}\left(h^{l}(X, Y), \xi\right)=g\left(A_{\xi}^{*} X, Y\right), \\
\bar{g}\left(h^{*}(X, P Y), N\right)=g\left(A_{N} X, P Y\right), \\
\bar{g}\left(h^{l}(X, \xi), \xi\right)=0, A_{\xi}^{*} \xi=0 .
\end{gathered}
$$

Definition 2.1 A $(2 n+1)$-dimensional Semi-Riemannian manifold $(\bar{M}, \bar{g})$ is called a contact metric manifold if there is a $(1,1)$ tensor field $\phi$, a vector field $V$, called the characteristic vector field, and its dual 1-form $\eta$ such that

$$
\begin{gathered}
\bar{g}(\phi X, \phi Y)=\bar{g}(X, Y)-\varepsilon \eta(X) \eta(Y), \bar{g}(V, V)=\varepsilon, \\
\phi^{2}(X)=-X+\eta(X) V, \bar{g}(X, V)=\varepsilon \eta(X), \\
d \eta(X, Y)=\bar{g}(X, \phi Y), \forall X, Y \in \Gamma(T M),
\end{gathered}
$$

where $\varepsilon= \pm 1$.

From the above definiton, it follows that

$$
\phi V=0, \eta \circ \phi=0, \eta(V)=1 .
$$

The $(\phi, V, \eta, \bar{g})$ is called a contact metric structure of $\bar{M}$. If $N_{\phi}+d \eta \otimes V=0$, we say that $\bar{M}$ has a normal contact structure, where $N_{\phi}$ is the Nijenhuis tensor field of $\phi$. A normal contact metric manifold is called a Sasakian manifold for which we have

$$
\begin{gathered}
\bar{\nabla}_{X} V=-\phi X . \\
\left(\bar{\nabla}_{X} \phi\right) Y=\bar{g}(X, Y) V-\varepsilon \eta(Y) X .
\end{gathered}
$$

Let $\left(M, g, S(T M), S\left(T M^{\perp}\right)\right)$ be a lightlike submanifold of $(\bar{M}, \bar{g})$. For any vector field $X$ tangent to $M$, we put

$$
\phi X=P X+Q X,
$$

where $P X$ and $Q X$ are the tangential and the transversal parts of $\phi X$, respectively.

Let's suppose $V$ is a spacelike vector field so that $\varepsilon=1$, it's similar when $V$ is a timelike vector field.

\section{Geodesic Invariant Lightlike Submanifolds}

Definition 3.1 Let $\left(M, g, S(T M), S\left(T M^{\perp}\right)\right)$ be a lightlike submanifold, tangent to the structure vector field $V, V \in S(T M)$, immersed in an indefinite Sasakian manifold $(\bar{M}, g)$, we say that $M$ is an invariant submanifolds of $\bar{M}$ if the following conditions are satisfied

$$
\phi(\operatorname{RadTM})=\operatorname{RadTM}, \phi(S(T M))=S(T M) .
$$

From (2.16), (2.17), (2.18) and (2.4) we have

$$
\begin{gathered}
h^{l}(X, V)=h^{s}(X, V)=0, \bar{\nabla}_{X} V=\nabla_{X} V=-P X, \quad(3.2) \\
h^{l}(X, \phi Y)=\phi h(X, Y)=h(\phi X, Y), \forall X, Y \in \Gamma(T M) .
\end{gathered}
$$

From (3.1) and (2.12) we have

$$
\phi \operatorname{ltr}(T M)=\operatorname{ltr}(T M), \phi\left(S\left(T M^{\perp}\right)\right)=S\left(T M^{\perp}\right) .
$$

Theorem 3.1 Let $\left(M, g, S(T M), S\left(T M^{\perp}\right)\right)$ be an invariant lightlike submanifold of an indefinite Sasakian manifold $\bar{M}$, then $M$ is totally geodesic if and only if $h^{l}$ and $h^{s}$ of $M$ are parallel.

Proof. Suppose $h^{l}$ is parallel, for any $X, Y, Z \in \Gamma(T M)$, we have

$$
\begin{aligned}
\left(\bar{\nabla}_{X} h^{l}\right)(Y, V)= & \bar{\nabla}_{X} h^{l}(Y, V)-h^{l}\left(\bar{\nabla}_{X} Y, V\right) \\
& -h^{l}\left(Y, \bar{\nabla}_{X} V\right)=0 .
\end{aligned}
$$

By (3.2), we have

$$
h^{l}(Y, V)=h^{l}\left(\bar{\nabla}_{X} Y, V\right)=0,
$$


so $h^{l}\left(Y, \bar{\nabla}_{X} Y\right)=0$. That is to say $h^{l}(Y, P X)=0$.

In a similar way, we can get $h^{s}(Y, P X)=0$. Thus, $M$ is totally geodesic.

Conversely, if $h^{l}(X, Y)=h^{s}(X, Y)=0$, since

$$
\begin{aligned}
\left(\bar{\nabla}_{X} h^{l}\right)(Y, Z)= & \bar{\nabla}_{X} h^{l}(Y, Z)-h^{l}\left(\bar{\nabla}_{X} Y, Z\right) \\
& -h^{l}\left(Y, \bar{\nabla}_{X} Z\right)=0, \\
\left(\bar{\nabla}_{X} h^{s}\right)(Y, Z)= & \bar{\nabla}_{X} h^{s}(Y, Z)-h^{s}\left(\bar{\nabla}_{X} Y, Z\right) \\
& -h^{s}\left(Y, \bar{\nabla}_{X} Z\right)=0,
\end{aligned}
$$

so $h^{l}$ and $h^{s}$ are parallel, which completes the proof.

\section{Geodesic Contact CR-Lightlike Submanifolds}

Definition 4.1 Let $\left(M, g, S(T M), S\left(T M^{\perp}\right)\right)$ be a lightlike submanifold, tangent to the structure vector field $V$, immersed in an indefinite Sasakian manifold $(\bar{M}, \bar{g})$. We say that $M$ is a contact $C R$-lightlike submanifold of $\bar{M}$ if the following conditions are satisfied [(A)] RadTM is a distribution on $M$ such that

$\operatorname{RadTM} \cap \phi(\operatorname{RadTM})=\{0\} .[(B)]$ There exist vector bundles $D_{0}$ and $D^{\prime}$ over $M$ such that

$$
\begin{gathered}
S(T M)=\left\{\phi(\operatorname{RadTM}) \oplus D^{\prime}\right\} \perp D_{0} \perp V, \\
\phi D_{0}=D_{0}, \phi D^{\prime}=L_{1} \perp L_{2},
\end{gathered}
$$

where $D_{0}$ is non-degenerate and $L_{1}=\operatorname{ltr}(T M), L_{2}$ is a vector subbundle of $S\left(T M^{\perp}\right)$. So we have the decomposition

$T M=\left\{D \perp \oplus D^{\prime}\right\} \perp V, D=\operatorname{RadTM} \perp \phi(\operatorname{RadTM}) \perp D_{0}$.

If we denote $\hat{D}=D \perp V$, then we have

$$
T M=\hat{D} \oplus D^{\prime}, \phi \hat{D}=\hat{D} .
$$

Definition 4.2 A contact CR-lightlike submanifold of an indefinite Sasakian manifold is called $\hat{D}$-geodesic contact CR-lightlike submanifold if its second fundamental form $h$ satisfied $h(X, Y)=0$, for any $X, Y \in \Gamma(\hat{D})$.

Definition 4.3 A contact CR-lightlike submanifold of an indefinite Sasakian manifold is called mixed geodesic contact CR-lightlike submanifold if its second fundamental form $h$ satisfied $h(X, Z)=0$, for any

$X \in \Gamma(\hat{D})$ and $Z \in \Gamma\left(D^{\prime}\right)$.

Definition 4.4 A contact CR-lightlike submanifold of an indefinite Sasakian manifold is called $D^{\prime}$-geodesic contact CR-lightlike submanifold if its second fundamental form $h$ satisfied $h(Z, U)=0$, for any $Z, U \in \Gamma\left(D^{\prime}\right)$.

Theorem 4.1 Let $M$ be a contact CR-lightlike submanifold of an indefinite Sasakian manifold $\bar{M}$.
Then $M$ is totally geodesic if and only if $\bar{g}\left(Y, A_{w} X\right)=\bar{g}\left(Y, D^{l}(X, W)\right), \nabla_{X} \phi Y$ has no components in $\phi L_{1}, Y \in \Gamma(T M-\operatorname{span}\{V\})$ or $X$ has no components in $\phi L_{1}$.

Proof. We know that $M$ is totally geodesic if and only if $h(X, Y)=0$, for any $X, Y \in \Gamma(T M)$. By the definition of the second fundamental form, $h(X, Y)=0$ is equivalent to $\bar{g}(h(X, Y), \xi)=0, \bar{g}(h(X, Y), W)=0$, for any $\xi \in \Gamma(\operatorname{RadTM}), W \in \Gamma\left(S\left(T M^{\perp}\right)\right)$.

From (2.4) and (2.7) we have

$$
\begin{aligned}
& \bar{g}(h(X, Y), \xi)=\bar{g}\left(\bar{\nabla}_{X} Y, \xi\right) \\
& =\bar{g}\left(\phi \bar{\nabla}_{X} Y, \phi \xi\right)+\eta\left(\bar{\nabla}_{X} Y\right) \eta(\xi) \\
& =\bar{g}\left(\phi \bar{\nabla}_{X} Y, \phi \xi\right) \\
& =\bar{g}\left(\bar{\nabla}_{X} \phi Y, \phi \xi\right)+\bar{g}(\bar{g}(X, Y) V+\eta(Y) X, \phi \xi) \\
& =\bar{g}\left(\nabla_{X} \phi Y, \phi \xi\right)+\eta(Y) \bar{g}(X, \phi \xi)
\end{aligned}
$$

and

$$
\begin{aligned}
& \bar{g}\left(h^{s}(X, Y), W\right)=\bar{g}\left(\bar{\nabla}_{X} Y, W\right) \\
& =X(\bar{g}(Y, W))-\bar{g}\left(Y, \bar{\nabla}_{X} W\right) \\
& =-\bar{g}\left(Y, \bar{\nabla}_{X} W\right) \\
& =-\bar{g}\left(Y,-A_{W} X+\nabla_{X}^{s} W+D^{l}(X, W)\right) \\
& =\bar{g}\left(Y, A_{W} X\right)-\bar{g}\left(Y, D^{l}(X, W)\right) .
\end{aligned}
$$

Thus, from (4.1) and (4.2), the proof is completed.

Theorem 4.2 Let $M$ be a contact CR-lightlike submanifold of an indefinite Sasakian manifold $\bar{M}$. Then $M$ is mixed geodesic if and only if $A_{\varphi Y} X$ has no components in $\phi \operatorname{RadTM} \perp L_{2}$.

Proof. By the definition, $M$ is mixed geodesic if and only if

$$
\begin{aligned}
& \bar{g}(h(X, Y), \xi)=0, \bar{g}(h(X, Y), W)=0 . \\
& \forall x \in \Gamma(\hat{D}), Y \in \Gamma\left(D^{\prime}\right) .
\end{aligned}
$$

Then we have

$$
\begin{aligned}
& \bar{g}(h(X, Y), \xi)=\bar{g}\left(\bar{\nabla}_{X} Y, \xi\right) \\
& =\bar{g}\left(\phi \bar{\nabla}_{X} Y, \phi \xi\right)+\eta\left(\bar{\nabla}_{X} Y\right) \eta(\xi) \\
& =\bar{g}\left(\phi \bar{\nabla}_{X} Y, \phi \xi\right) \\
& =\bar{g}\left(\bar{\nabla}_{X} \phi Y, \phi \xi\right)+\bar{g}(\bar{g}(X, Y) V+\eta(Y) X, \phi \xi) \\
& =\bar{g}\left(\bar{\nabla}_{X} \varphi Y, \phi \xi\right)+\eta(Y) \bar{g}(X, \phi \xi) \\
& =-\bar{g}\left(A_{\varphi Y} X, \phi \xi\right)+\eta(Y) \bar{g}(X, \phi \xi) \\
& =-\bar{g}\left(A_{\varphi Y} X, \phi \xi\right)
\end{aligned}
$$


and

$$
\begin{aligned}
& \bar{g}(h(X, Y), W)=\bar{g}\left(\bar{\nabla}_{X} Y, W\right) \\
& =\bar{g}\left(\phi \bar{\nabla}_{X} Y, \phi W\right)+\eta\left(\bar{\nabla}_{X} Y\right) \eta(W) \\
& =\bar{g}\left(\phi \bar{\nabla}_{X} Y, \phi W\right) \\
& =\bar{g}\left(\bar{\nabla}_{X} \phi Y, \phi W\right)+\bar{g}(\bar{g}(X, Y) V+\eta(Y) X, \phi W) \\
& =\bar{g}\left(\bar{\nabla}_{X} \phi Y, \phi W\right) \\
& =-\bar{g}\left(A_{\varphi Y} X, \phi W\right) .
\end{aligned}
$$

Thus, the proof of the theorem is complete.

Theorem 4.3 Let $M$ be a contact CR-lightlike submanifold of an indefinite Sasakian manifold $\bar{M}$. Then $M$ is $\hat{D}$-geodesic if and only if

$\nabla_{X}^{*} \phi \xi \in \Gamma\left(\phi \operatorname{RadTM} \perp \phi L_{2}\right), \quad \nabla_{X} Y$ has no components in $\phi L_{2}, \forall X, Y \in \Gamma(\hat{D})$.

Proof. $M$ is $\hat{D}$-geodesic if and only if $\bar{g}\left(h^{l}(X, Y), \xi\right)=0, \bar{g}\left(h^{s}(X, Y), W\right)=0$, for any $X, Y \in \Gamma(\hat{D}), \xi \in \Gamma(\operatorname{RadTM})$ and $W \in \Gamma\left(S\left(T M^{\perp}\right)\right)$.

Then we have

$$
\begin{aligned}
& \bar{g}(h(X, Y), \xi)=\bar{g}\left(\bar{\nabla}_{X} Y, \xi\right) \\
& =\bar{g}\left(\phi \bar{\nabla}_{X} Y, \phi \xi\right)+\eta\left(\bar{\nabla}_{X} Y\right) \eta(\xi) \\
& =\bar{g}\left(\phi \bar{\nabla}_{X} Y, \phi \xi\right) \\
& =\bar{g}\left(\bar{\nabla}_{X} \phi Y, \phi \xi\right)+\bar{g}(\bar{g}(X, Y) V+\eta(Y) X, \phi \xi) \\
& =\bar{g}\left(\bar{\nabla}_{X} \phi Y, \phi \xi\right) \\
& =-\bar{g}\left(\phi Y, \bar{\nabla}_{X} \phi \xi\right) \\
& =-\bar{g}\left(\phi Y, \nabla_{X}^{*} \phi \xi\right)
\end{aligned}
$$

and

$$
\begin{aligned}
& \bar{g}\left(h^{s}(X, Y), W\right)=\bar{g}\left(\bar{\nabla}_{X} Y, W\right) \\
& =\bar{g}\left(\phi \bar{\nabla}_{X} Y, \phi W\right)+\eta\left(\bar{\nabla}_{X} Y\right) \eta(W) \\
& =\bar{g}\left(\phi \bar{\nabla}_{X} Y, \phi W\right) \\
& =\bar{g}\left(\bar{\nabla}_{X} \phi Y, \phi W\right)+\bar{g}(\bar{g}(X, Y) V+\eta(Y) X, \phi W) \\
& =\bar{g}\left(\bar{\nabla}_{X} \phi Y, \phi W\right) \\
& =\bar{g}\left(\nabla_{X} Y, \phi W\right) .
\end{aligned}
$$

Thus the assertions of the theorem follows.

Theorem 4.4 Let $M$ be a contact CR-lightlike submanifold of an indefinite Sasakian manifold $\bar{M}$. Then $M$ is $D^{\prime}$-geodesic if and only if $A_{W} X, A_{\xi}^{*} X$ have no components in $\phi L_{2} \perp \phi(\operatorname{RadTM}) \forall X, Y \in \Gamma\left(D^{\prime}\right)$.

Proof. $M$ is $D^{\prime}$-geodesic if and, only if
$\bar{g}\left(h^{l}(X, Y), \xi\right)=0, \bar{g}\left(h^{s}(X, Y), W\right)=0$, for any

$X, Y \in \Gamma\left(D^{\prime}\right), \xi \in \Gamma(\operatorname{RadTM})$ and $W \in \Gamma\left(S\left(T M^{\perp}\right)\right)$.

So we have

$$
\begin{aligned}
\bar{g}(h(X, Y), \xi) & =\bar{g}\left(\bar{\nabla}_{X} Y, \xi\right)=-\bar{g}\left(Y, \bar{\nabla}_{X} \xi\right) \\
& =\bar{g}\left(A_{\xi}^{*} X, Y\right)
\end{aligned}
$$

and

$$
\begin{aligned}
\bar{g}(h(X, Y), W) & =\bar{g}\left(\bar{\nabla}_{X} Y, W\right)=-\bar{g}\left(Y, \bar{\nabla}_{X} W\right) \\
& =\bar{g}\left(A_{W} X, Y\right) .
\end{aligned}
$$

Thus the assertions of the theorem follows.

\section{Geodesic Contact SCR-Lightlike Submanifolds}

Definition 5.1 Let $\left(M, g, S(T M), S\left(T M^{\perp}\right)\right)$ be a lightlike submanifold, tangent to the structure vector field $V$, immersed in an indefinite Sasakian manifold $(\bar{M}, \bar{g})$. We say that $M$ is a contact SCR-lightlike submanifold of $\bar{M}$ if the following conditions are satisfied $[(A)]$ There exist real non-null distributions $D$ and $D^{\perp}$, such that

$$
\begin{aligned}
& S(T M)=D \perp D^{\perp} \perp V, \phi\left(D^{\perp}\right) \subset S\left(T M^{\perp}\right), \\
& D \cap D^{\perp}=\{0\},
\end{aligned}
$$

where $D^{\perp}$ is the orthogonal complementary to $D \perp V$ in $S(T M)$. [(B)]

$\phi D=D, \phi \operatorname{RadTM}=\operatorname{RadTM}, \phi \operatorname{lt}(T M)=\operatorname{ltr}(T M)$.

Hence we have the decomposition

$T M=\bar{D} \perp D^{\perp} \perp V, \bar{D}=D \perp \operatorname{RadTM}$.

Let us denote $\overline{\bar{D}}=\bar{D} \perp V$.

Definition 5.2 A contact SCR-lightlike submanifold of an indefinite Sasakian manifold is called mixed geodesic contact SCR-lightlike submanifold if its second fundamental form $h$ satisfied $h(X, Y)=0$, for any $X \in \Gamma(\bar{D})$ and $Y \in \Gamma\left(D^{\perp}\right)$.

Theorem 5.1 Let $M$ be a contact SCR-lightlike submanifold of an indefinite Sasakian manifold $\bar{M}$. Then $M$ is totally geodesic if and only if

$$
\begin{aligned}
& \left(L_{\xi} \bar{g}\right)(X, Y)=\left(L_{W} \bar{g}\right)(X, Y)=0, \forall X, Y \in \Gamma(T M), \\
& \xi \in \Gamma(\operatorname{Rad} T M), W \in \Gamma\left(S\left(T M^{\perp}\right)\right) .
\end{aligned}
$$

Proof. We know $M$ is totally geodesic if and only if

$$
\begin{aligned}
& \bar{g}(h(X, Y), \xi)=0, \bar{g}(h(X, Y), W)=0 . \\
& \forall X \in \Gamma(\hat{D}), Y \in \Gamma\left(D^{\prime}\right) .
\end{aligned}
$$


From (2.1) and Lie derivative we obtain

$$
\begin{aligned}
& \bar{g}(h(X, Y), \xi)=\bar{g}\left(\bar{\nabla}_{X} Y, \xi\right) \\
& =X(\bar{g}(Y, \xi))-\bar{g}(Y, \bar{\nabla}) X \xi \\
& =\bar{g}(Y,[\xi, X])-\bar{g}\left(Y, \bar{\nabla}_{\xi} X\right) \\
& =\bar{g}(Y,[\xi, X])-\xi(\bar{g}(X, Y))+\bar{g}\left(X, \bar{\nabla}_{\xi} Y\right) \\
& =\bar{g}(Y,[\xi, X])-\xi(\bar{g}(X, Y))+\bar{g}(X,[\xi, Y])+\bar{g}\left(\bar{\nabla}_{Y} \xi, X\right) \\
& =-\left(L_{\xi} \bar{g}\right)(X, Y)-\bar{g}\left(\xi, \bar{\nabla}_{Y} X\right) \\
& =-\left(L_{\xi} \bar{g}\right)(X, Y)-\bar{g}(h(X, Y), \xi) .
\end{aligned}
$$

Hence we have $2 \bar{g}(h(X, Y), \xi)=-\left(L_{\xi} \bar{g}\right)(X, Y)$. In a similar way, we can get

$$
2 \bar{g}(h(X, Y), W)=-\left(L_{W} \bar{g}\right)(X, Y),
$$

thus the proof is completed.

Theorem 5.2 Let $M$ be a contact SCR-lightlike submanifold of an indefinite Sasakian manifold $\bar{M}$. Then $M$ is mixed geodesic if and only if

$\nabla_{X}^{s} \phi Y \in \Gamma\left(D^{\perp}\right), A_{\phi Y} X \in \Gamma(\hat{\bar{D}})$, for any

$X \in \Gamma(\hat{\bar{D}}), Y \in \Gamma\left(D^{\perp}\right)$.

Proof. For any

$$
\begin{aligned}
& X \in \Gamma(\hat{\bar{D}}), Y \in \Gamma\left(D^{\perp}\right), \\
& \xi \in \Gamma(\operatorname{RadTM}), W \in \Gamma\left(S\left(T M^{\perp}\right)\right)
\end{aligned}
$$

denote by

$$
\phi X=P^{\prime} X+Q^{\prime} X, \phi W=B^{\prime} W+C^{\prime} W,
$$

where $P^{\prime} X \in \Gamma(\bar{D}), Q^{\prime} X \in \Gamma\left(\phi D^{\perp}\right), B^{\prime} W \in \Gamma\left(D^{\perp}\right)$ and $C^{\prime} W \in \Gamma\left(S\left(T M^{\perp}\right)-\phi D^{\perp}\right)$.

If $M$ is mixed geodesic, then $h(X, Y)=\bar{\nabla}_{X} Y-\nabla_{X} Y=0$. From the definition, there exists $W \in \Gamma\left(S\left(T M^{\perp}\right)\right)$ such that $\phi W=Y$. Thus we have

$$
\begin{aligned}
& 0=\bar{\nabla}_{X} \phi W-\nabla_{X} Y=\phi \bar{\nabla}_{X} W-\nabla_{X} Y \\
& =\phi\left(-A_{W} X+\nabla_{X}^{t} W\right)-\nabla_{X} Y \\
& =-P^{\prime} A_{W} X-Q^{\prime} A_{W} X+B^{\prime} \nabla_{X}^{t} W+C^{\prime} \nabla_{X}^{t} W-\nabla_{X} Y .
\end{aligned}
$$

From the definition of the $Q^{\prime}$ and $C^{\prime}$, we know that $Q^{\prime} A_{W} X=C^{\prime} \nabla_{X}^{t} W=0$. So we have

$\nabla_{X}^{t} W \in \Gamma\left(\phi D^{\perp}\right), A_{W} X \in \Gamma(\hat{\bar{D}})$. From $\phi W=Y$ and

(2.13), we have $W=-\phi Y$, thus the proof is completed.

Theorem 5.3 Let $M$ be a contact SCR-lightlike submanifold of an indefinite Sasakian manifold $\bar{M}$. Then $D^{\perp}$ defines a totally geodesic foliation if and only if $h^{s}(X, \phi Z)$ and $h^{s}(X, \phi N)$ has no components in $\Gamma\left(\phi\left(D^{\perp}\right)\right), \forall X \in \Gamma\left(D^{\perp}\right), Z \in \Gamma(\bar{D})$.

Proof. From the definition, we have that $D^{\perp}$ is a totally geodesic foliation if and only if $\nabla_{X} Y \in \Gamma\left(D^{\perp}\right)$, for any $X, Y \in \Gamma\left(D^{\perp}\right)$, which is equivalent to

$$
\begin{aligned}
& g\left(\nabla_{X} Y, Z\right)=g\left(\nabla_{X} Y, N\right)=0, \\
& \forall Z \in \Gamma(\bar{D}), N \in \Gamma(\operatorname{ltr}(T M)) .
\end{aligned}
$$

Then we have

$$
\begin{aligned}
& g\left(\nabla_{X} Y, Z\right)=\bar{g}\left(\bar{\nabla}_{X} Y, Z\right)=-\bar{g}\left(Y, \bar{\nabla}_{X} Z\right) \\
& =-\bar{g}\left(\phi Y, \phi \bar{\nabla}_{X} Z\right)-\eta(Y) \eta\left(\bar{\nabla}_{X} Z\right) \\
& =-\bar{g}\left(\phi Y, \phi \bar{\nabla}_{X} Z\right) \\
& =-\bar{g}\left(\phi Y, \bar{\nabla}_{X} \phi Z+g(X, Z) V+\eta(Z) X\right) \\
& =-\bar{g}\left(\phi Y, \bar{\nabla}_{X} \phi Z\right) \\
& =-\bar{g}\left(\phi Y, h^{s}(X, \phi Z)\right)
\end{aligned}
$$

and

$$
\begin{aligned}
& g\left(\nabla_{X} Y, N\right)=\bar{g}\left(\bar{\nabla}_{X} Y, N\right) \\
= & \bar{g}\left(\phi \bar{\nabla}_{X} Y, \phi N\right)+\eta\left(\bar{\nabla}_{X} Y\right) \eta(N) \\
= & \bar{g}\left(\phi \bar{\nabla}_{X} Y, \phi N\right) \\
= & \bar{g}\left(\bar{\nabla}_{X} \phi Y+g(X, Y) V+\eta(Y) X, \phi N\right) \\
= & \bar{g}\left(\bar{\nabla}_{X} \phi Y, \phi N\right) \\
= & -\bar{g}\left(\phi Y, \bar{\nabla}_{X} \phi N\right) \\
= & -\bar{g}\left(\phi Y, h^{s}(X, \phi N)\right) .
\end{aligned}
$$

Thus the assertion is proved.

\section{References}

[1] D. N. Kupeli, “Singular Semi-Riemannian Geometry,” Kluwer, Dordrecht, 1996.

[2] K. L. Duggal and A. Bejancu, "Lightlike Submanifolds of Semi-Riemannian Manifolds and Applications," Kluwer Academic, Dordrecht, 1996.

[3] B. Sahin, "Transversal Lightlike Submanifolds of Indefinite Kaehler Manifolds,” Analele Universitaii de Vest, Timisoara Seria Matematica-Informatica, Vol. 44, No. 1, 2006, pp. 119-145.

[4] B. Sahin and R. Günes, "Geodesic CR-Lightlike Submanifolds," Contributions to Algebra and Geometry, Vol. 42, No. 2, 2001, pp. 583-594. 
[5] K. L. Duggal and B. Sahin, "Lightlike Submanifolds of Indefinite Sasakian Manifolds," International Journal of Mathematics and Mathematical Sciences, Article ID 57585, 2007, 21 Pages.
[6] K. L. Duggal and B. Sahin, "Generalized Cauchy-Rieman Lightlike Submanifolds of Indefinite Sasakian Manifolds,” Acta Mathematica Hungarica, Vol. 122, No. 1-2, 2009, pp. 45-58. doi:10.1007/s10474-008-7221-8 